\title{
Mechanical thrombectomy for acute ischaemic stroke during therapeutic anticoagulation: long-term outcomes
}

\author{
Klaudia Nowak ${ }^{1}$, Ewa Wlodarczyk ${ }^{2}$, Karolina Porębska ${ }^{1}$, Żaneta Chatys-Bogacka $^{1}$, Jeremiasz Jagiełła ${ }^{1}$, \\ Roman Pułyk ${ }^{2}$, Joanna Słowik ${ }^{3}$, Tadeusz Popiela ${ }^{4}$, Agnieszka Słowik ${ }^{1}$ \\ ${ }^{1}$ Department of Neurology, Jagiellonian University in Krakow, Poland \\ ${ }^{2}$ Department of Neurology, University Hospital in Krakow, Poland \\ ${ }^{3}$ Department of Dental Prophylaxis and Experimental Dentistry, Jagiellonian University Medical College, Poland \\ ${ }^{4}$ Department of Radiology, University Hospital in Krakow, Poland
}

\begin{abstract}
:
Aim of study. Mechanical thrombectomy (MT) is one of the aetiological treatment options recommended for anticoagulated patients with acute ischaemic stroke (AIS). We analysed its long-term outcomes using the modified Rankin Score (mRS) or mortality on day 90 .

Clinical rationale for the study. Data describing the anticoagulant efficacy and safety of MT in patients with AIS is limited.

Materials and methods. This study included 291 patients with AIS (49\% women, mean [SD] age 66 [15] years) who underwent MT in the Comprehensive Stroke Centre in Krakow, Poland. Data describing demographics, stroke risk factors, NIHSS on admission, postprocedural thrombolysis in cerebral infarction score, 24-hour postprocedural haemorrhagic transformation (ECASS-2) as seen on computed tomography, and time between stroke onset and groin puncture was collected. The outcome measure was the mRS on day 90 after stroke onset (a favourable outcome was defined as an mRS not exceeding 2 points; an unfavourable outcome was death).
\end{abstract}

Results. Thirty-seven patients (13\%) were on therapeutic anticoagulation during MT. Univariate analysis showed that anticoagulated patients were older and more likely to have been diagnosed with hypertension, ischaemic heart disease, or atrial fibrillation. The patient groups did not differ in terms of clot location, postprocedural thrombolysis in cerebral infarction score, haemorrhagic transformation on computed tomography, or $\mathrm{mRS}$ on day 90 . Multivariate logistic regression analysis showed that younger age, male sex, no history of diabetes mellitus, lower NIHSS score on admission, shorter time between stroke onset and groin puncture, and better recanalisation were associated with favourable outcomes at day 90 , and that therapeutic anticoagulation was not (OR, $1.00 ; 95 \% \mathrm{Cl}, 0.46-2.15 ; \mathrm{p}=0.99)$. Anticoagulation did not affect mortality at day $90(\mathrm{OR}, 1.28 ; 95 \% \mathrm{Cl}, 0.56-2.92 ; \mathrm{p}=0.55)$.

Conclusion and clinical implications. In anticoagulated patients with AIS, MT does not affect long-term outcomes.

Key words: mechanical thrombectomy, acute ischaemic stroke, novel oral anticoagulants, anticoagulation, atrial fibrillation (Neurol Neurochir Pol 2020; 54 (6): 538-543)

\section{Introduction}

Atrial fibrillation (AF) substantially contributes to stroke risk [1]. Both vitamin $\mathrm{K}$ antagonists (VKAs) and novel oral anticoagulants (NOACs) significantly decrease this risk.

However, $1.11-3.24 \%$ of patients with AF develop stroke despite anticoagulant treatment [2].
A couple of aetiological treatment options are recommended for anticoagulated patients who have developed acute ischaemic stroke (AIS):

1. an intravenous tissue plasminogen activator when treatment includes a VKA, and the international normalised ratio (INR) is less than or equal to 1.7 , or when treatment includes a NOAC and laboratory tests show that the 
anticoagulatory effect of NOACs is probably absent or when the last dose of the NOAC was more than 48 hours previously, or

2. mechanical thrombectomy (MT) when the patient fulfills all commonly accepted treatment guidelines $[3,4]$.

\section{Clinical rationale for study}

Data describing the efficacy and safety of MT for AIS in patients using oral anticoagulants is limited.

\section{Materials and methods}

\section{Experimental procedure}

This retrospective analysis was performed on prospectively collected data from the Krakow Stroke Data Bank, a single-centre, hospital-based registry of clinical radiological and genetic data from patients with AIS, systematically collected since 2007. This study design was approved by the Jagiellonian University Ethical Committee (KBET 54/B/2007). The analysis included data from 291 patients with AIS treated with MT between January 2013 and April 2019. Detailed patient work-ups are described elsewhere [5]. Participants were categorised as either those given therapeutic anticoagulation or those not anticoagulated before stroke. The former were treated with full doses of oral anticoagulants (i.e. VKAs, NOACs, or low-molecular-weight heparin $[\mathrm{LMWH}]$ ) with or without haemostasis disturbances detected via laboratory tests at admission. Contemporaneous anticoagulant treatment information was collected from medical histories and information from the patients or relatives. The latter were those with medical histories or relatives not describing anticoagulant treatments and without haemostasis disturbances detected via laboratory tests at admission. The outcome measure was mRS on day 90 after stroke onset. A favourable outcome was defined as an $\mathrm{mRS}$ not exceeding 2 points.

\section{Statistical methods}

Clinical characteristics and outcomes were compared using the unpaired $t$ test or the chi-square test, as appropriate. To identify potential independent outcome predictors, variables showing $\mathrm{p}$-values $<0.05$ in univariate analyses were subsequently included in a multivariate regression analysis. Because this study was focused on analysing the significance of therapeutic anticoagulation on long-term outcome measures, two models for each outcome were used: one with, and one without, therapeutic anticoagulation.

All statistical analyses were performed using Statistica software (version 12.5, Statsoft, Poland) When $\mathrm{p}<0.05$, statistical significance was recognised.

\section{Results}

The group receiving anticoagulant therapy comprised 37 patients (13\%). Of these, 14 were treated with VKAs, four with LMWH, and 19 with NOACs (six with dabigatran, and 13 with rivaroxaban). Therapeutic anticoagulation was used to treat atrial fibrillation in 30 patients and venous thromboembolism in seven patients. Compared to those not receiving anticoagulants, these patients were older and more likely to have ischaemic heart disease or AF (Tab. 1). Clot locations were similar in both groups, as were postprocedural thrombolysis in cerebral infarction (TICI) scores, 24-hour postprocedural haemorrhagic transformations based on computed tomography (CT) according to ECASS-2 [6], and mRS distributions at day 90 (Tab. 1). Cardioembolic stroke was more common in anticoagulated patients, but large vessel disease stroke, stroke of unknown cause, and stroke of rare aetiology were not (Tab. 1).

Favourable outcomes were found at day 90 for 154 patients (52.9\%); 63 (21.6\%) died. Results of univariate analyses comparing patients with favourable to those with unfavourable outcomes, and comparing survivors to non-survivors at day 90, are set out in Table 2.

Multivariate logistic regression analysis showed that younger age, male sex, no history of diabetes mellitus, lower NIHSS score on admission, better recanalisation as measured by TICI score ( $2 \mathrm{~b}$ or 3 ), and shorter time from stroke onset to groin puncture were associated with favourable outcomes (an mRS not exceeding 2 at day 90). Therapeutic anticoagulation did not affect outcome (Tab. 3). In one multivariate logistic regression model, older age and poor recanalisation (TICI scores of 0,1 , or $2 \mathrm{a}$ ), but not therapeutic anticoagulation, were significantly correlated with mortality at day 90 (Tab. 3).

\section{Discussion}

The number of AIS patients treated by means of IVT or MT has increased significantly in recent years [7-9]. As shown in other studies [10-16], we found that MT used to treat AIS in anticoagulated patients did not change long-term outcomes compared to non-coagulated AIS patients treated by MT. Age, sex, time from stroke onset to groin puncture, neurological deficit as measured by the NIHSS, rate of recanalisation as assessed using the TICI score, and diabetes mellitus were confirmed as correlating with outcome.

However, it should be noted that outcome based on mRS on day 90 was defined differently in other studies. An excellent outcome has been described as an mRS of 0 or 1 [17], a favourable outcome has been described as an mRS of 0,1 , or $2[10-12,14,17]$, and an unfavourable outcome has been defined as an mRS of 3-6, 4-6, or $6[10,13-16,18]$. Of nine published studies, five, including this one [10,13, 14], evaluated at least two outcome measures based on mRS.

It is well known that MT is included in the guidelines for treating AIS [3, 4], and that today it is the most effective treatment related to good outcomes. The number of points needed to treat is 3 to achieve an mRS of 0,1 , or 2 on day 90 [19]. It should be noted, however, that most studies on this topic show 
Table 1. Demographics, clinical characteristics and outcomes in patients under therapeutic anticoagulation and in patients not anticoagulated at time of mechanical thrombectomy (univariate analysis)

\begin{tabular}{|c|c|c|c|}
\hline Parameter & $\begin{array}{l}\text { Anticoagulated } \\
\text { patients }(n=37)\end{array}$ & $\begin{array}{l}\text { Non-anticoagulated } \\
\text { patients }(n=254)\end{array}$ & $\mathbf{p}$ \\
\hline \multicolumn{4}{|l|}{ Demographics } \\
\hline Age, years (mean $\pm S D$ ) & $71.8( \pm 8.5)$ & $65.8( \pm 14.9)$ & 0.0001 \\
\hline Female $(n, \%)$ & $18(48.6)$ & $125(49.2)$ & 0.95 \\
\hline \multicolumn{4}{|l|}{ Stroke risk factors } \\
\hline Hypertension ( $\mathrm{n}, \%)$ & $33(89.2)$ & $176(69.5)$ & 0.012 \\
\hline Ischaemic heart disease $(n, \%)$ & $19(51.4)$ & $70(27.7)$ & 0.0033 \\
\hline Atrial fibrillation (n, \%) & $30(81.0)$ & $64(25.4)$ & $<0.00001$ \\
\hline Diabetes mellitus $(n, \%)$ & $10(27.0)$ & $86(34.0)$ & 0.41 \\
\hline \multicolumn{4}{|l|}{ Clinical parameters } \\
\hline Time from stroke onset to groin puncture (hours) (mean \pm SD) & $4.07 \pm 1.39$ & $4.53 \pm 1.17$ & 0,13 \\
\hline NIHSS on admission (mean \pm SD) & $14.2 \pm 6.0$ & $15.7 \pm 6.2$ & 0.19 \\
\hline \multicolumn{4}{|l|}{ Clot location } \\
\hline MCA, M1 (n, \%) & $17(45.9)$ & $123(48.4)$ & 0.78 \\
\hline MCA, M2 (n, \%) & $6(16.2)$ & $43(16.9)$ & 0.91 \\
\hline MCA, M3 $(n, \%)$ & $1(2.7)$ & $6(2.3)$ & 0.90 \\
\hline$M 1+M 2(n, \%)$ & $0(0.0)$ & $2(1.0)$ & $\mathrm{N} / \mathrm{A}$ \\
\hline ICA $(n, \%)$ & $6(16.2)$ & $43(16.9)$ & 0.91 \\
\hline $\mathrm{ICA}+\mathrm{MCA}, \mathrm{M} 1(\mathrm{n}, \%)$ & $4(10.8)$ & $12(4.7)$ & 0.13 \\
\hline $\mathrm{A} 1(\mathrm{n}, \%)$ & $0(0.0)$ & $0(0.0)$ & N/A \\
\hline$B C A$ and/or VA $(n, \%)$ & $2(5.3)$ & $21(8.3)$ & 0.55 \\
\hline Multiple locations $(n, \%)$ & $1(2.7)$ & $3(1.2)$ & 0.46 \\
\hline \multicolumn{4}{|l|}{ TICl score post-thrombectomy } \\
\hline $\mathrm{TICl} 0(\mathrm{n}, \%)$ & $3(8.1)$ & $19(7.5)$ & 0.86 \\
\hline $\operatorname{TICl} 1(n, \%)$ & $3(8.1)$ & $12(4.7)$ & \\
\hline $\mathrm{TICl} 2 \mathrm{a}(\mathrm{n}, \%)$ & $5(13.5)$ & $36(14.2)$ & \\
\hline $\mathrm{TICl} 2 \mathrm{~b}(\mathrm{n}, \%)$ & $7(18.9)$ & $64(25.2)$ & \\
\hline $\mathrm{TICl} 3(\mathrm{n}, \%)$ & $19(51.4)$ & $123(48.4)$ & \\
\hline \multicolumn{4}{|l|}{ Haemorrhagic transformation (ECASS-2) } \\
\hline No haemorrhage $(n, \%)$ & $19(51.4)$ & $147(57.9)$ & 0.16 \\
\hline $\mathrm{HI}-1(\mathrm{n}, \%)$ & $16(43.2)$ & $70(27.6)$ & \\
\hline $\mathrm{HI}-2(\mathrm{n}, \%)$ & $0(0.0)$ & $1(0.4)$ & \\
\hline $\mathrm{PH}-1(\mathrm{n}, \%)$ & $1(2.7)$ & $15(5.9)$ & \\
\hline $\mathrm{PH}-2(\mathrm{n}, \%)$ & $1(2.7)$ & $21(8.3)$ & \\
\hline \multicolumn{4}{|l|}{ Stroke aetiology } \\
\hline Large vessel disease ( $n, \%)$ & $2(5.4)$ & $29(11.5)$ & $<0.05$ \\
\hline Small vessel disease $(n, \%)$ & $0(0.0)$ & $0(0.0)$ & \\
\hline Cardiac-embolic stroke $(n, \%)$ & $31(83.8)$ & $104(41.3)$ & \\
\hline Unknown cause $(n, \%)$ & $3(8.1)$ & $72(28.2)$ & \\
\hline Rare cause $(n, \%)$ & $1(2.7)$ & $50(19.8)$ & \\
\hline \multicolumn{4}{|l|}{ Stroke outcome (modified Rankin Score on day 90) } \\
\hline mRS: 0 & $14(37.9)$ & $87(34.3)$ & 0.89 \\
\hline mRS: 1 & $2(5.4)$ & $34(13.4)$ & \\
\hline $\mathrm{mRS}: 2$ & $3(8.1)$ & $14(5.5)$ & \\
\hline $\mathrm{mRS}: 3$ & $2(5.4)$ & $23(9.1)$ & \\
\hline $\mathrm{mRS}: 4$ & $3(8.1)$ & $20(7.9)$ & \\
\hline mRS: 5 & $3(8.1)$ & $23(9.0)$ & \\
\hline $\mathrm{mRS}: 6$ & $10(27.0)$ & $53(20.8)$ & \\
\hline
\end{tabular}

BCA - basal cerebral artery; ECASS-2 classification: $\mathrm{HI}-1:$ small petechiae, $\mathrm{HI}-2$ : more confluent petechiae, $\mathrm{PH}-1:<30 \%$ of the infarcted area with mild space-occupying effect, $\mathrm{PH}-2>30 \%$ of the infarcted area with significant space-occupying effect; ICA - internal carotid artery; MCA - middle cerebral artery, MCA M1 - sphenoidal or horizontal segment; MCA M2 - insular segment; MCA M3 - opercular segment; N/A - not applicable; NS - not significant; $\mathrm{SD}$ - standard deviation; $\mathrm{TCCl}$ - Thrombolysis in Cerebral Infarction: $\mathrm{TICI} 0$ - no perfusion; $\mathrm{TCCl} 1$ - penetration with minimal perfusion; $\mathrm{TCCl} 2 \mathrm{a}$ - only partial filling (less than two-thirds) of the entire vascular territory is visualised; $\mathrm{TCC} 2 \mathrm{~b}$ - complete filling of all of the expected vascular territory is visualised but filling is slower than normal; $\mathrm{TICl} 3-$ complete perfusion; $\mathrm{VA}$ - vertebral arteries 
Table 2. Demographics and stroke characteristics in patients treated by mechanical thrombectomy with mRS: 0-2 vs. mRS: 3-6 and mRS: 0-5 vs. mRS: 6 at day 90 after stroke (univariate analysis)

\begin{tabular}{|c|c|c|c|c|c|c|}
\hline Parameter & $\begin{array}{c}\text { mRS: } 0-2 \\
N=154\end{array}$ & $\begin{array}{c}\text { mRS: } 3-6 \\
N=137\end{array}$ & $\mathbf{p}$ & $\begin{array}{c}\text { mRS: } 0-5 \\
N=228\end{array}$ & $\begin{array}{l}\text { mRS: } 6 \\
N=63\end{array}$ & $\mathbf{p}$ \\
\hline \multicolumn{7}{|l|}{ Demographics } \\
\hline Age (years), mean $\pm S D$ & $62.9 \pm 14.7$ & $70.7 \pm 12.9$ & 0.000002 & $65.4 \pm 14.7$ & $71.1 \pm 12.4$ & 0.0051 \\
\hline Gender (females), n (\%) & $61(39.6)$ & $82(59.9)$ & 0.00057 & $108(47.4)$ & $35(55.6)$ & 0.25 \\
\hline \multicolumn{7}{|l|}{ Stroke risk factors } \\
\hline Hypertension, n (\%) & $106(68.8)$ & $103(75.2)$ & 0.39 & $158(69.3)$ & $51(81)$ & 0.069 \\
\hline Diabetes mellitus, n (\%) & $30(19.5)$ & $50(36.5)$ & 0.0012 & $58(25.4)$ & $22(34.9)$ & 0.14 \\
\hline Ischaemic heart disease, n (\%) & $42(27.3)$ & $41(29.9)$ & 0.62 & $60(26.3)$ & $23(36.5)$ & 0.11 \\
\hline Atrial fibrillation, $\mathrm{n}(\%)$ & $55(35.7)$ & $61(44.5)$ & 0.13 & $87(38.2)$ & $29(46)$ & 0.26 \\
\hline \multicolumn{7}{|l|}{ Clinical parameters } \\
\hline Poor recanalisation $\mathrm{TICl}, \mathrm{n}(\%) \quad 0-2 \mathrm{a}$ & $20(13.0)$ & $58(42.3)$ & $<0.00001$ & $52(22.4)$ & $27(42.9)$ & 0.0012 \\
\hline $\begin{array}{l}\text { Time from stroke onset to groin puncture (hours), mean } \pm \\
\text { SD }\end{array}$ & $4.3 \pm 1.7$ & $4.7 \pm 1.8$ & 0.02 & $4.4 \pm 1.81$ & $4.54 \pm 1.44$ & 0.75 \\
\hline NIHSS on admission, mean \pm SD & $14.5 \pm 6.5$ & $16.6 \pm 5.6$ & 0.0027 & $15.1 \pm 6.1$ & $16.9 \pm 6.4$ & 0.04 \\
\hline \multicolumn{7}{|l|}{ Treatment } \\
\hline Therapeutic anticoagulation during acute ischaemic stroke & $19(12.3)$ & $18(13.2)$ & 0.84 & $27(11.8)$ & $10(15.8)$ & 0.4 \\
\hline
\end{tabular}

mRS — modified Rankin Scale; NIHSS — National Institutes of Health Stroke Scale; TICI — thrombolysis in cerebral infarction

that MT does not decrease mortality [20]. Additional studies should focus on the various aspects of MT prognoses and on two general outcome measures: favourable and unfavourable.

The number of published studies analysing the significance of MT in anticoagulated patients with stroke is increasing. Taken together, 1,013 anticoagulated patients treated with MT have been studied, and of those 176 (17.4\%) were given NOACs. An analysis of 28 patients with AIS who were enrolled in the Registry of Acute Ischaemic Stroke Under New Oral Anticoagulants, anticoagulated with NOACs, and treated using MT was performed by Purrucker et al. [10] without a control group. Separately, Seiffge et al. [16] used a propensity score matching method to compare three cohorts of acute stroke patients: 78 anticoagulated with NOACs, 441 with VKAs, and 8,938 not anticoagulated.

In neither study were NOACs found to have a negative influence on the safety and outcomes of acute stroke treated by MT.

To determine whether anticoagulation in patients with AIS treated by MT was significant, several national and international registries have been recently announced: the RASUNOA-prime (Rationale and design of the Registry of Acute Stroke Under Novel Oral Anticoagulants-prime) registry [21], the ENDOSTROKE registry [12], and the Registry of Acute Ischaemic Stroke Under New Oral Anticoagulants [10].

Our study also showed that MT in anticoagulated patients did not increase the risk of intracranial haemorrhage as observed on CT scans performed within 24 hours of the procedure. All studies, including this one, show similar distributions of brain haemorrhage severity according to ECASS-II in anticoagulated and non-anticoagulated patients $[10-16,17,18]$.

In our study, all patients with AIS who were current users of anticoagulants were treated with MT without prior intravenous thrombolysis. Of four patients receiving full doses of LMWH, two had prolonged APTTs. Similarly, of 14 receiving VKAs, eight had prolonged INRs. Of six patients receiving dabigatran, two had prolonged APTTs, and 11/13 taking rivaroxaban had prolonged PTs. 23 patients or relatives were unable to indicate when the last dose of anticoagulant had been administered.

Currently, specific coagulation tests analysing NOAC concentrations are not commonly available. The test that is typically used requires an hour to yield results, hampering treatment decisions about anticoagulation in acute stroke patients. Because about $10 \%$ of acute stroke patients admitted to a stroke unit are anticoagulated, bedside tests delivering immediate results on anticoagulation status are needed.

This study has several limitations. Firstly, its small sample size can lead to type II errors. Secondly, it carries all limitations inherent in retrospective analyses of prospectively collected data. Thirdly, because the numbers of patients receiving each specific anticoagulant were small, we were unable to compare patient groups.

\section{Clinical implications and future directions}

This study confirms the results of others [22,23], namely that MT is safe and efficacious in anticoagulated patients with AIS. 
Table 3. Multivariate regression models analysing factors affecting favourable outcome $(m R S=0-2)$ or death $(m R S=6)$ at day 90 including and excluding therapeutic anticoagulation

\begin{tabular}{|c|c|c|c|}
\hline \multicolumn{4}{|c|}{ Factors affecting favourable outcome at day 90 (mRS:0-2) } \\
\hline & OR & $95 \% \mathrm{Cl}$ & $\mathbf{P}$ \\
\hline \multicolumn{4}{|l|}{ Demographics } \\
\hline Age (years) & 0.95 & $0.93-0.98$ & 0.000081 \\
\hline Gender (female) & 0.57 & $0.32-0.998$ & 0.048 \\
\hline \multicolumn{4}{|l|}{ Stroke risk factors } \\
\hline Diabetes mellitus & 0.42 & $0.22-0.77$ & 0.0053 \\
\hline \multicolumn{4}{|l|}{ Clinical parameters } \\
\hline NIHSS on admission & 0.95 & $0.91-0.99$ & 0.031 \\
\hline Poor recanalisation (TICl post-thrombectomy: $0,1,2 \mathrm{a}$ ) & 0.15 & $0.08-0.30$ & 0.000000029 \\
\hline Time from stroke onset to groin puncture, hours & 0.74 & $0.62-0.88$ & 0.00051 \\
\hline \multicolumn{4}{|c|}{ Factors affecting favourable outcome at day 90 (mRS:0-2), model including therapeutic anticoagulation } \\
\hline \multicolumn{4}{|l|}{ Demographics } \\
\hline Age (years) & 0.96 & $0.93-0.98$ & 0.000093 \\
\hline Gender (female) & 0.57 & $0.32-0.999$ & 0.049 \\
\hline \multicolumn{4}{|l|}{ Stroke risk factors } \\
\hline Diabetes mellitus & 0.42 & $0.22-0.77$ & 0.0053 \\
\hline \multicolumn{4}{|l|}{ Clinical parameters } \\
\hline NIHSS on admission & 0.95 & $0.91-0.99$ & 0.032 \\
\hline Poor recanalisation (TICl post-thrombectomy: 0, 1, 2a) & 0.15 & $0.08-0.30$ & 0.000000029 \\
\hline Time from stroke onset to groin puncture & 0.74 & $0.62-0.88$ & 0.00053 \\
\hline \multicolumn{4}{|l|}{ Treatment } \\
\hline Therapeutic anticoagulation & 1.00 & $0.46-2.15$ & 0.99 \\
\hline \multicolumn{4}{|c|}{ Factor affecting risk of death at day 90} \\
\hline \multicolumn{4}{|l|}{ Demographics } \\
\hline Age (years) & 1.03 & $1.008-1.06$ & 0.0077 \\
\hline Gender (female) & 0.97 & $0.52-1.81$ & 0.91 \\
\hline \multicolumn{4}{|l|}{ Clinical parameters } \\
\hline Poor recanalisation (TICl post-thrombectomy: 0, 1, 2a) & 2.69 & $1.46-4.95$ & 0.0014 \\
\hline NIHSS & 1.04 & $0.99-1.10$ & 0.094 \\
\hline Factors affecting risk of & coagu & & \\
\hline \multicolumn{4}{|l|}{ Demographics } \\
\hline Age (years) & 1.03 & $1.01-1.06$ & 0.0099 \\
\hline Gender (female) & 0.98 & $0.52-1.83$ & 0.95 \\
\hline \multicolumn{4}{|l|}{ Clinical parameters } \\
\hline Poor recanalisation (TICl post-thrombectomy: 0, 1, 2a) & 2.68 & $1.45-4.93$ & 0.0015 \\
\hline NIHSS & 1.04 & $0.99-1.10$ & 0.086 \\
\hline \multicolumn{4}{|l|}{ Treatment } \\
\hline Therapeutic anticoagulation & 1.28 & $0.56-2.92$ & 0.55 \\
\hline
\end{tabular}

mRS - modified Rankin Scale; NIHSS - National Institutes of Health Stroke Scale; TICI - thrombolysis in cerebral infarction

However, systematic studies that include data on coagulation status and the decision-making procedure before MT, and comparing the various anticoagulation drugs in the context of acute stroke treatment, are necessary.
Acknowledgements: We received no grant from any funding agency in the public, commercial, or not-for-profit sector to support this research.

Disclosures: The authors declare no conflict of interest. 


\section{References:}

1. Freedman B, Porpara TS, Lip GY. Stroke prevention in atrial fibrillation. Lancet. 2016; 388: 806-17.

2. Alberts M, Eikelboom J, Hankey G. Antithrombotic therapy for stroke prevention in non-valvular atrial fibrillation. The Lancet Neurology. 2012; 11(12): 1066-1081, doi: 10.1016/s1474-4422(12)70258-2.

3. Powers WJ, Rabinstein AA, Ackerson T, et al. 2018 Guidelines for the Early Management of Patients With Acute Ischemic Stroke: A Guideline for Healthcare Professionals From the American Heart Association/American Stroke Association. Journal of Vascular Surgery. 2018; 67(6): 1934, doi: 10.1016/j.jvs.2018.04.007.

4. Correction to: Guidelines for the Early Management of Patients With Acute Ischemic Stroke: 2019 Update to the 2018 Guidelines for the Early Management of Acute Ischemic Stroke: A Guideline for Healthcare Professionals From the American Heart Association/American Stroke Association. Stroke. 2019; 50(12): e440-e441, doi: 10.1161/ STR.0000000000000215, indexed in Pubmed: 31765293.

5. Nowak K, Derbisz J, Jagiełła J, et al. Time from stroke onset to groin puncture affects rate of recanalisation after mechanical thrombectomy: a real-life single centre experience. Neurol Neurochir Pol. 2020; 54(2): 156-160, doi: 10.5603/PJNNS.a2020.0024, indexed in Pubmed: 32242914.

6. Yaghi S, Willey JZ, Cucchiara B, et al. American Heart Association Stroke Council; Council on Cardiovascular and Stroke Nursing; Council on Clinical Cardiology; and Council on Quality of Care and Outcomes Research. Treatment and Outcome of Hemorrhagic Transformation After Intravenous Alteplase in Acute Ischemic Stroke: A Scientific Statement for Healthcare Professionals From the American Heart Association/ American Stroke Association. Stroke. 2017; 48(12): e343-e361, doi: 10.1161/STR.0000000000000152, indexed in Pubmed: 29097489.

7. Lattanzi S, Silvestrini M. Future challenges of stroke treatment. Neurol Neurochir Pol. 2018; 52(3): 415-416, doi: 10.1016/j. pjnns.2018.02.004, indexed in Pubmed: 29486885.

8. Łabuz-Roszak B, Starostka-Tatar A, Lasek-Bal A, et al. Diagnostics, treatment and secondary prevention of ischemic stroke in the Silesian Province, Poland between 2009 and 2015. Neurol Neurochir Pol. 2018; 52(2): 235-242, doi: 10.1016/j.pjnns.2017.11.003, indexed in Pubmed: 29198761.

9. Wiącek M, Kaczorowski R, Sieczkowski B, et al. Mechanical thrombectomy: Determining the proportion of eligible acute ischemic stroke patients in the cohort of single academic stroke center. Neurol Neurochir Pol. 2018; 52(3): 359-363, doi: 10.1016/j.pjnns.2017.12.010, indexed in Pubmed: 29331206.

10. Purrucker JC, Wolf M, Haas K, et al. Safety of Endovascular Thrombectomy in Patients Receiving Non-Vitamin K Antagonist Oral Anticoagulants. Stroke. 2016; 47(4): 1127-1130, doi: 10.1161/STROKEAHA.116.012684, indexed in Pubmed: 26931156.

11. Zapata-Wainberg G, Ximénez-Carrillo Á, Trillo S, et al. Madrid Stroke Network. Mechanical thrombectomy in orally anticoagulated patients with acute ischemic stroke. J Neurointerv Surg. 2018; 10(9): 834838, doi: 10.1136/neurintsurg-2017-013504, indexed in Pubmed: 29275325.
12. Uphaus T, Singer OC, Berkefeld J, et al. Safety of endovascular treatment in acute stroke patients taking oral anticoagulants. Int J Stroke. 2017; 12(4): 412-415, doi: 10.1177/1747493016677986, indexed in Pubmed: 28353412.

13. De Marchis GM, Jung S, Colucci G, et al. Intracranial hemorrhage, outcome, and mortality after intra-arterial therapy for acute ischemic stroke in patients under oral anticoagulants. Stroke. 2011; 42(11): 3061-3066, doi: 10.1161/STROKEAHA.111.615476, indexed in Pubmed: 21980194.

14. Benavente L, Larrosa D, García-Cabo C, et al. Safety and Efficacy of Mechanical Thrombectomy in Acute Ischemic Stroke of Anticoagulated Patients-A Prospective Observational Study. J Stroke Cerebrovasc Dis. 2016; 25(9): 2093-2098, doi: 10.1016/j.jstrokecerebrovasdis.2016.06.006, indexed in Pubmed: 27378732.

15. Rebello LC, Haussen DC, Belagaje S, et al. Endovascular Treatment for Acute Ischemic Stroke in the Setting of Anticoagulation. Stroke. 2015; 46(12): 3536-3539, doi: 10.1161/STROKEAHA.115.011285, indexed in Pubmed: 26470775.

16. Seiffge DJ, Hooff RJ, Nolte CH, et al. NOACISP Study Group*. Recanalization therapies in acute ischemic stroke patients: impact of prior treatment with novel oral anticoagulants on bleeding complications and outcome. Circulation. 2015; 132(13): 1261-1269, doi: 10.1161/ CIRCULATIONAHA.115.015484, indexed in Pubmed: 26232277.

17. Wong JWP, Churilov L, Dowling R, et al. Safety of Endovascular Thrombectomy for Acute Ischaemic Stroke in Anticoagulated Patients Ineligible for Intravenous Thrombolysis. Cerebrovasc Dis. 2018; 46(5-6): 193-199, doi: 10.1159/000493801, indexed in Pubmed: 30384367.

18. Kurowski D, Jonczak K, Shah Q, et al. Safety of Endovascular Intervention for Stroke on Therapeutic Anticoagulation: Multicenter Cohort Study and Meta-Analysis. J Stroke Cerebrovasc Dis. 2017; 26(5): 1104-1109, doi: 10.1016/j.jstrokecerebrovasdis.2016.12.027, indexed in Pubmed: 28110890.

19. Endovascular Therapy for Ischemic Stroke. New England Journal of Medicine. 2015; 372(24): 2363-2366, doi: 10.1056/nejmc1504715.

20. Campbell BCV, Mitchell PJ, Kleinig TJ, et al. EXTEND-IA Investigators. Endovascular therapy for ischemic stroke with perfusion-imaging selection. N Engl J Med. 2015; 372(11): 1009-1018, doi: 10.1056/ NEJMoa1414792, indexed in Pubmed: 25671797.

21. Haas K, Purrucker JC, Rizos T, et al. Rationale and design of the Registry of Acute Stroke Under Novel Oral Anticoagulants-prime (RASUNOA-prime). Eur Stroke J. 2019; 4(2): 181-188, doi: 10.1177/2396987318812644, indexed in Pubmed: 31259266.

22. Meinel TR, Frey S, Arnold M, et al. Clinical presentation, diagnostic findings and management of cerebral ischemic events in patients on treatment with non-vitamin $\mathrm{K}$ antagonist oral anticoagulants - A systematic review. PLoS One. 2019; 14(3): e0213379, doi: 10.1371/ journal.pone.0213379, indexed in Pubmed: 30925155.

23. Liu M, Zheng Y, Li G. Safety of Recanalization Therapy in Patients with Acute Ischemic Stroke Under Anticoagulation: A Systematic Review and Meta-Analysis. J Stroke Cerebrovasc Dis. 2018; 27(9): 22962305, doi: 10.1016/j.jstrokecerebrovasdis.2018.04.012, indexed in Pubmed: 30017747. 